\title{
Spatial Cognition and the Brain
}

\author{
NeIl Burgess \\ Institute of Cognitive Neuroscience, University College London, United Kingdom
}

\begin{abstract}
Recent advances in the understanding of spatial cognition are reviewed, focusing on memory for locations in large-scale space and on those advances inspired by single-unit recording and lesion studies in animals. Spatial memory appears to be supported by multiple parallel representations, including egocentric and allocentric representations, and those updated to accommodate selfmotion. The effects of these representations can be dissociated behaviorally, developmentally, and in terms of their neural bases. It is now becoming possible to construct a mechanistic neural-level model of at least some aspects of spatial memory and imagery, with the hippocampus and medial temporal lobe providing allocentric environmental representations, the parietal lobe egocentric representations, and the retrosplenial cortex and parieto-occipital sulcus allowing both types of representation to interact. Insights from this model include a common mechanism for the construction of spatial scenes in the service of both imagery and episodic retrieval and a role for the remainder of Papez's circuit in orienting the viewpoint used. In addition, it appears that hippocampal and striatal systems process different aspects of environmental layout (boundaries and local landmarks, respectively) and do so using different learning rules (incidental learning and associative reinforcement, respectively).
\end{abstract}

Key words: parietal; hippocampal; striatal; fMRI; place cells; grid cells; allocentric; egocentric; computational modeling

\section{Introduction}

The explosion of interest in cognitive neuroscience derives from the ability of recent advances in neuroscience to shed new light on the mechanisms supporting cognition. Within this endeavor, the field of spatial cognition is particularly well placed due to the ease with which similar (spatial) experimental paradigms can be applied to both humans and animals. Thus neuroscientific findings in animals can be integrated with noninvasive (behavioral, neuropsychological, and neuroimaging) findings in humans. Accordingly, recent years have seen the reasonably direct application of insights from single-unit recording in freely moving animals to understanding the mechanisms of human cognition regarding memory for locations within largescale space.

One theme to emerge, upon which I elaborate in this article, is the growing acceptance within the field of human spatial cognition of a long-held tenet of animal neuroscience (e.g., White \& McDonald 2002) that multiple parallel systems are at play, making use of a variety of reference frames. Thus the emphasis of

Address for correspondence: Neil Burgess, Institute of Cognitive Neuroscience, University College London, 17 Queen Square, London WC1N 3AR, U.K

n.burgess@ucl.ac.uk research has shifted from exclusive comparisons, for example, "Is spatial memory egocentric?" (Wang \& Spelke 2002; Shelton \& McNamara 1997), to addressing how the various systems combine to support behavior under different circumstances (Burgess 2006; Mou et al. 2004).

There has also been considerable recent progress in identifying the specific characteristics of neural representations of spatial location, including the startling recent discovery of an entirely new type of spatial representation "grid cells" (Hafting et al. 2005). These new findings are, in turn, beginning to feedback into cognitive models of spatial behavior. A crucial aid in relating neurophysiological findings to behavior is the use of computational modeling, which I touch upon where appropriate.

In this review I focus on the basic representations and mechanisms supporting spatial memory, navigation, and imagery. This necessarily leads to a focus on posterior parts of the brain: parietal, retrosplenial, and medial temporal neocortex as well as the hippocampal and striatal systems. Prefrontal cortex is only briefly mentioned, in the context of supplying simulated motor efference in the service of planning/imagining potential movements and in strategic mediation between hippocampal and striatal systems in controlling behavior. The review is structured according to different types of representations and processes and the 
corresponding neural systems. I start at the more sensory end, with egocentric representations of location and their updating to accommodate self-motion, and move on to more abstract allocentric representations of location and their updating to accommodate selfmotion. These issues, their neural bases, and their relationship to memory and imagery are treated within the framework of a model of medial temporal-parietal interactions in spatial cognition (Byrne et al. 2007; Burgess et al. 2001b; Becker \& Burgess 2001). The second part of the review concerns the relationships between hippocampal and striatal systems in spatial memory and navigation: which aspects of environmental information they process; which learning rules they use; and how they combine to control behavior. The discussion focuses on the application of insights from spatial paradigms to more general issues such as the idea of a "geometric module" in the brain and the roles played by the hippocampal, parietal, and striatal systems in supporting declarative and procedural memory and imagery.

\section{Multiple Parallel Reference Frames for Location}

\section{Egocentric Representations}

Locations in the external world can be represented in a variety of ways. Sensory information is generally acquired in the coordinates of the corresponding receptor (e.g., retinotopic for vision and head-centered for audition), while actions must be specified in the appropriate body-centered coordinates for the corresponding effector. Sensorimotor integration, as in reaching for a visual target, requires translation between these various egocentric representations. Evidence for all of these egocentric representations can be found at the level of single neurons in sensory and motor cortices, and mechanisms for translation between them are suggested by "gain field" responses of neurons recorded in posterior parietal area $7 \mathrm{a}$ in primates. These neurons respond to visual stimuli at a specific retinotopic location, but their rate of firing is also modulated by the orientation of the monkey's gaze relative to the head (Andersen et al. 1987), by the orientation of the head relative to the trunk, or by the orientation of the monkey within the testing room (Snyder et al. 1998), see FIGURE 1. These gain-field responses are ideal for translating locations between the various egocentric reference frames (Zipser \& Andersen 1988; Pouget \& Sejnowski 1997; Deneve et al. 2001).

Of particular interest for the following sections, area $7 \mathrm{a}$ is the posterior parietal area most strongly connected with the medial temporal lobe, and the neurons there whose firing is modulated by the orientation of the monkey within the room can support translation between egocentric and allocentric representations of locations. These neurons potentially allow translation between allocentric representations of environmental layout in the medial temporal lobe and head-centered representations required for imagining spatial scenes in medial parietal areas, see below and (Byrne et al. 2007; Burgess et al. 2001b). In addition, the anterior bank of the parieto-occipital sulcus, which runs between the medial parietal and retrosplenial cortices, contains visually responsive neurons which respond to stimuli presented at a given location irrespective of the direction of gaze (Galletti et al. 1995).

Behavioral evidence for egocentric representations in human spatial memory includes "alignment effects" in retrieval of an array of objects that was studied from a specific viewpoint. Thus the time taken to correctly recognize the array in photographs from other viewpoints around the array increases with the size of the angular difference of the test viewpoint from the encoding viewpoint (see, e.g., Diwadkar \& McNamara 1997). Equally, if people are asked to close their eyes and imagine being at a different location and orientation and then to point to where an object in the array would be (tasks sometimes referred to as judgments of relative direction), they are faster and more accurate when the imagined viewpoint has the same direction as the studied viewpoint (Shelton \& McNamara 1997). These findings are consistent with storage of a viewpoint-dependent representation of the array, followed by a cumulative process of mental movement of viewpoint. (Note, however, that some findings can also be interpreted as interference between the imagined perspective and the participant's current perspective, rather than the stored perspective, e.g., May 2004).

\section{Self-Motion and Egocentric Spatial Updating}

An interesting puzzle implied by egocentric perceptual representations is that of the perceived stability of the external world despite the rapid and rapidly changing motion of our sensory receptors as we move. The problem of perceptual stability is beyond the scope of this review (see Ross et al. 2001; Bridgeman et al. 1994; Melcher 2007 for more on visual stability). Suffice it to say, following Helmholtz (1866), that a major component of the solution appears to be the automatic updating of sensory representations by information about intended movements, often referred to as "motor efference copy." In a recent parallel to the long history of behavioral studies of visual stability, electrophysiological studies in primate posterior parietal cortex have examined the effects of eye movements 
A

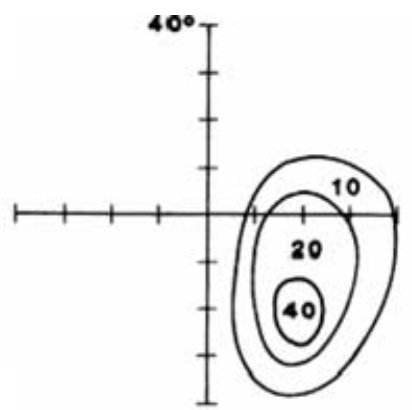

B

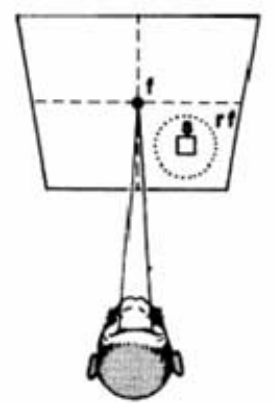

FIx center

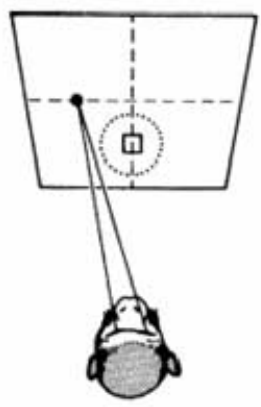

FIx loft

C

All otIm. rotinal $(20,-20)$

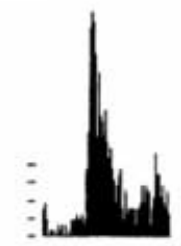

$F \mid x:(-20,20)$
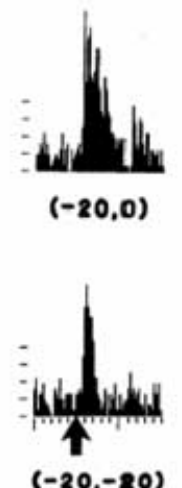
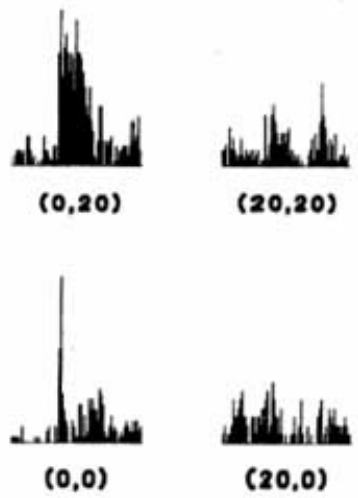

D
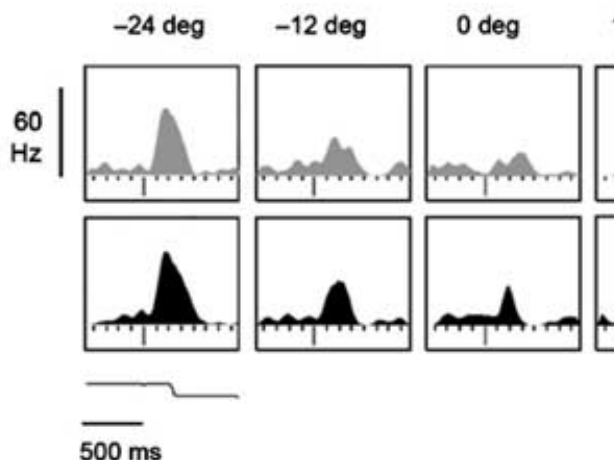

$12 \mathrm{deg}$
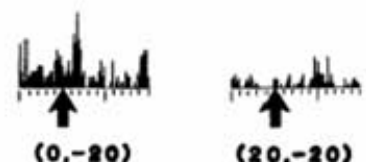

$(20,-20)$

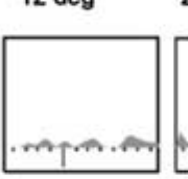

$24 \mathrm{deg}$
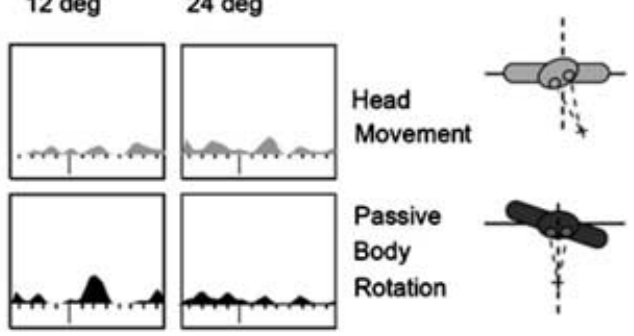

$500 \mathrm{~ms}$

FIGURE 1. Parietal gain fields: encoding of the retinotopic location of a stimulus modulated by the angle of gaze relative to the head (B-C), the angle of the head relative to the trunk (D), or the angle of the trunk in the lab (not shown). (A) Example of a neuron in primate parietal area $7 \mathrm{a}$ with a retinotopic receptive field for visual stimuli, showing peak firing rate as a function of stimulus location relative to fixation at the center. (B) The angle of gaze relative to the head can be varied, modulating the amplitude of the response to a stimulus in the same retinotopic location. (C) Arrow indicates stimulus onset; the position of each plot and figures in brackets indicates the angle of fixation. (D) Retinotopic responses can also be modulated by the angle of the head relative to the trunk. Some neurons show the same modulation by angle for movement of the head or for passive rotation of the trunk (shown, cf. above and below). Others show modulation by the angle of gaze within the room for the same angles of gaze relative to the head and of the head relative to the trunk. Adapted from Andersen, Essick, \& Siegel (1985) and Snyder, Grieve, Brotchie, \& Andersen (1998).

on the responses of single neurons. Interestingly, neurons with retinotopic receptive fields, for example, in the lateral intraparietal area, can be seen to update their responses so as to respond to stimuli which will be in the receptive field after a saccadic eye movement, even though the stimulus has actually disappeared before the completion of the movement. These results indicate spatial updating of neuronal representations by motor efference copy (see e.g., Colby \& Goldberg 1999 for a review).

Of course, the other potential mechanisms for spatial updating, aside from motor efference copy, should not be ignored. These include the integration of vestibular signals reflecting accelerations of the head, 
proprioceptive information regarding actual movements performed, and optic flow. All of these types of information can contribute to the processes of spatial updating and have been extensively studied within the more restricted context of "path integration": updating a representation of one's own displacement from the start of a movement trajectory by integrating the velocities experienced along the trajectory. We initially consider the likely egocentric processes supporting spatial updating in parietal areas and later consider likely allocentric processes supporting spatial updating in medial temporal areas. Note that, although spatial updating and path integration are regarded as egocentric processes in some treatments (e.g., Wang \& Spelke 2002), categorization of these processes per se is arbitrary, depending on whether the object or start locations are updated relative to the participant or whether the location of the participant is updated relative to the locations of objects, start positions, or other aspects of the environment.

Following work from Rieser (1989) and others, Simons and Wang (Simons \& Wang 1998; Wang \& Simons 1999) introduced an elegant paradigm for dissociating the contributions to spatial memory of sensory-bound representations such as "visual snapshots" from representations of locations relative to the body which are automatically updated to accommodate self-motion. In this paradigm, people see five objects on a circular table and later have to indicate which one has been moved. In between presentation and test phases, with the table out of view, it is possible to have the person change his or her viewpoint, or for the table to be rotated. In four conditions, either the person's viewpoint or the table is rotated about the center of the table or both are rotated, or neither. The angle of rotation is the same for the viewpoint and for the table so that, if both are rotated, the egocentric locations of the objects relative to the viewer are unchanged. See FIgURE 2 conditions _, P, PT, and $\mathrm{T}$. The consequence of these manipulations is a $2 \times 2$ factorial design in which the test array is either consistent or inconsistent with viewpoint-dependent representations of locations (consistent if both viewpoint and table are rotated together or if neither move) and also consistent or inconsistent with representations that are updated by self-motion (consistent if the viewpoint alone changes or if neither move). The results indicate a strong positive effect of consistency with representations updated by self-motion and a weaker effect of consistency with viewpoint-dependent sensory representations.

Another paradigm for investigating the presence of egocentric or allocentric representations in spatial memory concerns the distribution of errors in pointing to object locations, developed by Wang and Spelke (2000). In this paradigm, the participant views an array of objects scattered throughout a room and must then point to them from within a chamber in the center of the room (from which the objects can not longer be seen). Wang and Spelke reasoned that disorientating the participant by blindfolded rotation would have different effects upon egocentric and allocentric representations of the object locations. Namely, updating of individual egocentric representations will induce different amounts of error across the different locations, while updating of an integrated allocentric representation should induce similar amounts of error across the different locations. Consistent with their egocentric-only model (Wang \& Spelke 2002), Wang and Spelke (2000) found that blindfolded rotation increased the variance in errors across object locations.

An interesting parallel to the effects of actual selfmotion on spatial representations is provided by studies of imagined movement of viewpoint. In experiments on imagery, subjects study an array of objects and are subsequently blindfolded. They can then be asked to indicate the locations of objects following either imagined rotation of the array of objects or following imagined translocation of themselves to a new viewpoint around the array. In these situations performance is superior following imagined movement of the viewer than following an equivalent imagined movement of the array (e.g., Wraga et al. 2000). It is only when the array consists of a single location that performance for imagined array-rotation approaches that for imagined movement of the viewer.

Thus, there is strong evidence that egocentric representations of locations are maintained in the brain and that these are automatically updated by our own movements, intentions to move, or imagined movements. However, it is also possible that, where multiple locations or extended layouts are concerned, it is more efficient to maintain a cognitive representation of the world and to update our own location within it, rather than maintaining multiple egocentric representations each of which is affected differently by self-motion. In addition, having to retain information over prolonged self-motion increases the importance of knowledge of locations relative to environmental landmarks in avoiding the cumulative errors associated with egocentric updating. As we shall see in the next section, recent evidence points to the presence of these allocentric or world-centered representations in parallel to the egocentric representations discussed above. 
A

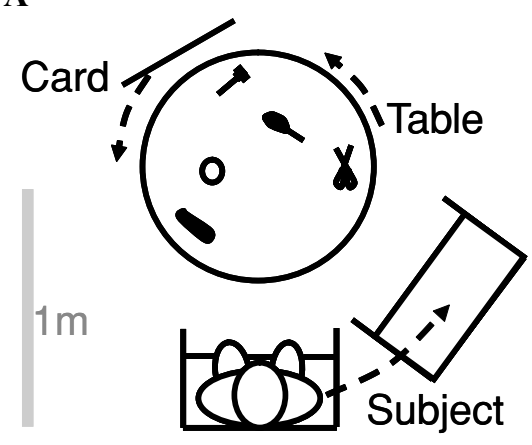

B

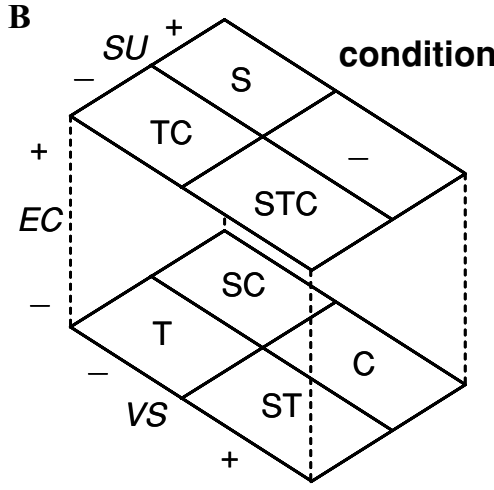

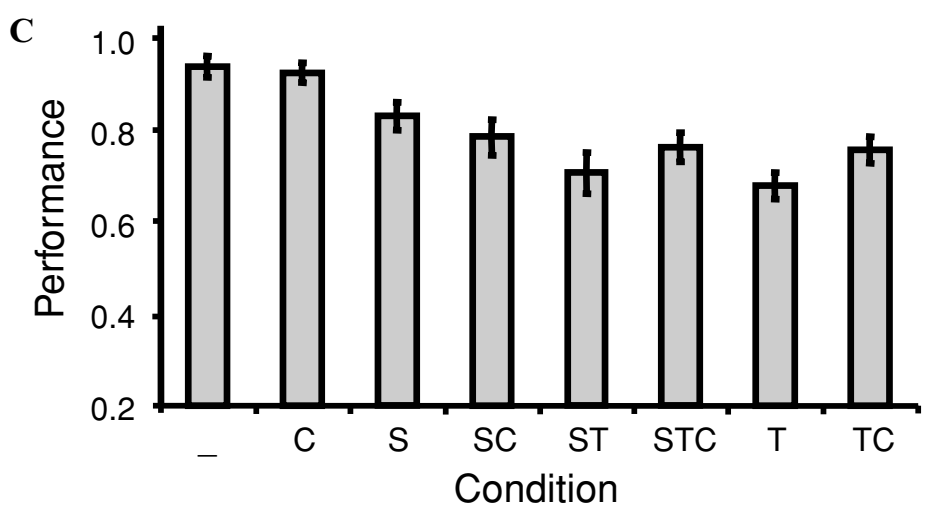

FIGURE 2. Paradigm for dissociating spatial reference frames, based on Simons and Wang (1998). (A) After initially seeing the array of objects and before being asked which one has moved, the position of the person $(P)$, the table $(T)$, or the external cue card $(C)$ can be rotated about the center of the table. (B) These changes provide a fully factorial manipulation of the consistency (+ or - ) of the test array with 3 different potential types of stored representation: visual snapshots (VS), representations spatially updated to accommodate the subject's movement (SU), representations of location relative to the external cue card (EC). (C) Performance benefits from consistency with any of these representations: the more the test array is consistent, the better performance is. Adapted from Burgess, Spiers, \& Paleologou (2004).

\section{Allocentric Representations}

Although egocentric representations provide the obvious format for sensory perception and motor action, and transformations between such egocentric representations suffice for short-term sensorimotor integration, it has long been argued that memory over the longer term is likely better served by allocentric representations centered on environmental landmarks (e.g., Milner et al. 1999; O'Keefe \& Nadel 1978). Updating of egocentric representations of location to accommodate self-motion (referred to as "path integration" when the location in question is the start of the path) will fall prey to cumulative error after relatively short paths (e.g., Etienne et al. 1996). Thus, when attempting to return to a previously visited location from a new direction after more than a few minutes of self-motion, representation of the location relative to the available environmental landmarks will often be of more use than egocentric representations. See Burgess (2006) for further discussion.

The idea of parallel representations (egocentric versus allocentric in the above discussion) with differential utility according to the amount of self-motion between presentation and test was recently investigated by Waller and Hodgson (2006). They reinterpreted the results of Wang and Spelke (2000), described above, arguing that increased variance in pointing errors after blindfolded rotation might result from a switch from using an accurate but transient representation to using a less accurate but more integrated enduring representation. In support of their interpretation, they found that, although variation in pointing errors increases 
A

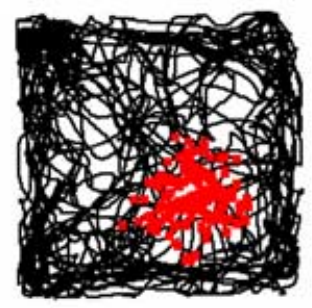

B

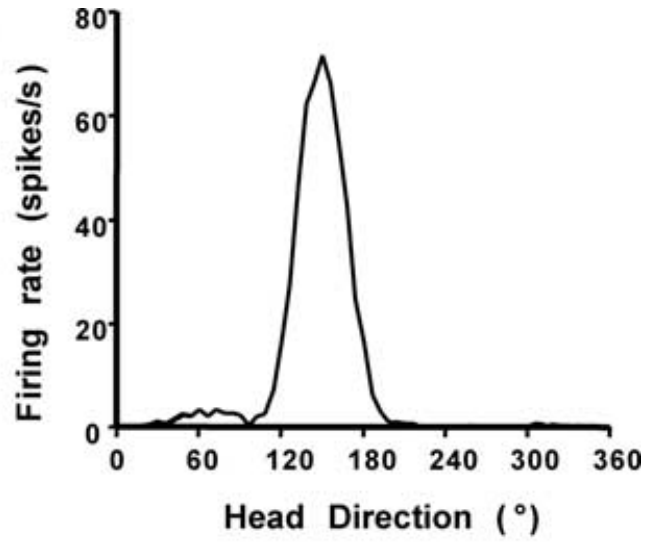

C

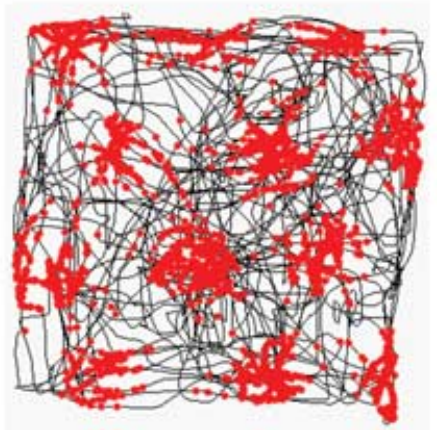

D

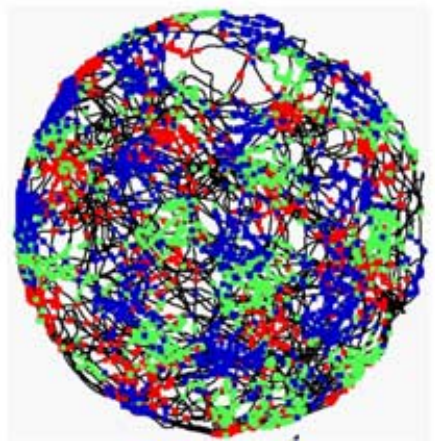

FIGURE 3. Place cells, head-direction cells, and grid cells. (A) Example of a place cell: the black line shows the rat's path in exploring a square enclosure, red squares show the locations at which a single neuron fired an action potential. (B) Example of a head-direction cell, showing firing rate as a function of head direction within the enclosure. (C) Example of a grid cell: firing occurs at an array of locations arranged in a regular triangular grid across the environment (same color scheme as A). (D) Example of three neighboring grid cells simultaneously recorded on the same tetrode, action potentials from the three cells shown in red, blue, and green. Adapted from Jeffery \& Burgess (2006).

after disorientation (consistent with the use of a less accurate representation), variation in the errors made when judging the relative direction of one object from another actually reduced after disorientation (consistent with the use of a more integrated representation). Waller and Hodgson also found that the effect of "disorientation" on pointing error occurs in an all-or-none fashion after rotations of $135^{\circ}$ or more, consistent with a switch from one representation to the other after movements of a certain magnitude. In addition, they found no disorientation-related increase in pointing error variation when pointing to objects within a very familiar environment, consistent with the development of more accurate enduring representations with exposure to an environment, and preferential use of them even over short movements and timescales. Here I interpret Waller and Hodgson's "enduring representation" as likely to be allocentric, see Burgess (2006) for further discussion. Below I briefly outline some of the neurophysiological evidence for allocentric representations in the mammalian brain and then describe some recent experiments indicating the presence of allocentric representations in human spatial cognition.

A neural representation of the animal's location relative to the surrounding environment can be seen in the firing of "place cells" in the hippocampus of rats (O'Keefe 1976) and primates (Ono et al. 1991), see Muller (1996) for a review. This representation is supported by representations of orientation (Taube 1998) and a grid-like representation suitable for path integration (Hafting et al. 2005) in nearby regions, both also environment centered. See FIGURE 3. The orientation of the firing patterns of these cells is primarily determined by distal visual cues, when available, with place cells specifically encoding location relative to extended boundaries in the environment, given this orientation (O'Keefe \& Burgess 1996; Hartley et al. 2000). These representations appear to guide 
behavior in spatial memory paradigms in which simple egocentric representations do not suffice. In these situations, behavioral responses match the firing of the cells (Kubie et al. 2007; Lenck-Santini et al. 2005; O'Keefe \& Speakman 1987). In addition, hippocampal lesions or inactivations impair performance in these tasks (e.g., Morris et al. 1982; Packard \& McGaugh 1996).

Recent investigations of the neural bases of navigation in humans have made use of desk-top virtual reality $(\mathrm{VR})$ : allowing simulation of movement through large-scale space in stationary subjects, albeit without the vestibular and proprioceptive components. Using VR, neural responses resembling those of place cells have been found in the human brain, clustered in the hippocampus (Ekstrom et al. 2003), while functional neuroimaging (Maguire et al. 1998a; Hartley et al. 2003; Iaria et al. 2003) and neuropsychological (Abrahams et al. 1997; Spiers et al. 2001a; Spiers et al. 2001b) data confirm the involvement of the human hippocampus in accurate large-scale navigation. In addition, Hartley and colleagues (2004) used VR to investigate the effect of deformation of the environmental boundary on human search locations, finding results compatible with the assumption that place cells guide behavior, given how place cells respond to such manipulations (O'Keefe \& Burgess 1996).

Direct evidence for allocentric representations in human spatial cognition has come from recent paradigms designed to replicate earlier experiments showing evidence of egocentric representations (discussed above), but also designed to probe any allocentric representations which might exist in parallel. Thus, memory for locations within an array has recently been found to show effects of the alignment of the testing perspective with directions defined by aspects of the external environment, as well as with those defined by the person's initial viewpoint (Mou \& McNamara 2002; Schmidt \& Lee 2006). When an array of objects contains an intrinsic axis (e.g., defined by symmetry), improved performance is found when pointing to objects from imagined viewpoints that are aligned with this axis or aligned with environmental features such as testing room walls (Mou \& McNamara 2002; Schmidt \& Lee 2006) and external landmarks (McNamara et al. 2003).

The use of allocentric and egocentric representations can also be dissociated within Simons and Wang's (1998) egocentric spatial updating paradigm (see above). In the original paradigm the conditions consistent with representations updated by self-motion, which all involve object locations that remain station- ary within the testing room, are also consistent with allocentric representations centered on environmental cues. Thus, some of the effect ascribed to spatial updating may be due to the presence of allocentric representations. To pull apart these multiple influences, Burgess et al. (2004) included independent manipulation of environmental cues: testing effects of consistency with viewpoint-dependent, spatially updated, and allocentric representations within a $2 \times 2 \times 2$ design, see FIGURE 2. In this test, people viewed an array of fluorescent objects with an external fluorescent cue, in darkness, and subsequently indicated which object had moved. Between presentation and test, the person's viewpoint, the array, or the external cue could be rotated to change the consistency of the test array with either type of representation. In addition to replicating the effects of consistency with viewpoint-dependent and spatially updated representations (when the cue did not move), an effect of consistency with the orientation of the external cue was also found. For example, performance increased when the card and table moved together compared to when one or other moved alone. Thus, allocentric representations of object locations relative to environmental cues probably exist in parallel to egocentric representations of location relative to the subject.

The Simons and Wang-inspired paradigm of dissociating frames of reference by shifting the viewpoint and/or the array of objects has recently been successfully applied to developmental psychology. Thus, representations of locations within the testing room appear to be present as early as 3 years and to make a greater contribution to behavior than egocentric snapshots at this age (Nardini et al. 2006). Representations of location relative to the intrinsic frame of reference of the array appear to develop between years three and six. Although the relative dependence of room-related responding on allocentric representations or egocentric spatial updating is not clear, the results demand a rethink of Piagetian ideas of early egocentrism at least (Piaget \& Inhelder 1956).

\section{Temporo-Parietal Mechanisms of Spatial Memory, Imagery, and Motion-Related Updating}

The nature of the representation of location by place cells has received much study over the several decades since their discovery. As a result, a neural-level model of spatial memory has begun to emerge from these findings, in combination with findings in related areas and in the parietal lobe. As a starting point, we briefly review some of the evidence concerning how environmental cues determine the spatial firing fields 

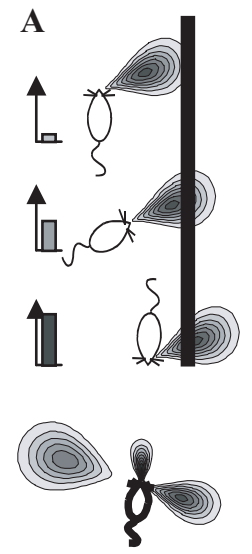

B
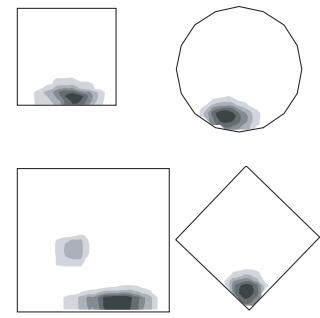

Data

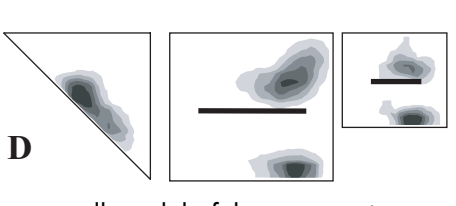

C
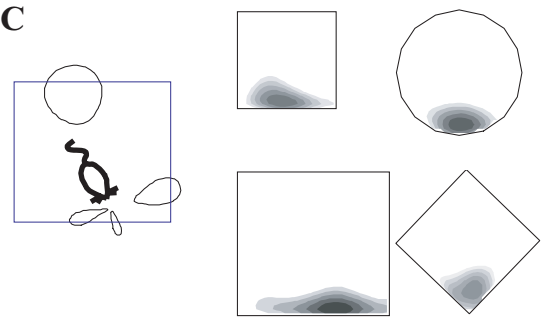

Model
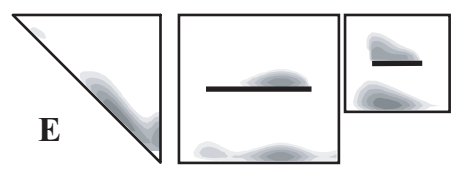

FIGURE 4. The boundary vector cell model of the sensory input to place cells. (A) Putative place cell inputs (boundary vector cells, BVCs) are assumed to be tuned to respond to the presence of a boundary at a given distance and allocentric direction (firing rate indicated by the bar on the left), with broader tuning at greater distances (below). (B) The firing of a place cell in four different enclosures can be modeled by an appropriate selection of BVC inputs. (C) The model can predict the firing of the cell in new configurations of the enclosure, including those with an internal barrier. (E) The actual firing of the cell in these environments reasonably matches the prediction. (D) Adapted from Burgess \& Hartley (2002).

of place cells, and thus contribute to the rat's sense of self-location.

The relative independence of place cell firing from low-level sensory representations can be seen in the independence of place cell firing from the animal's orientation as it explores open environments (Muller \& Kubie 1989) and in the robustness of the response to removal of the sensory cues controlling the orientation of the firing fields within the environment (O'Keefe \& Conway 1978; O’Keefe \& Speakman 1987; Pico et al. 1985; Fenton et al. 2000). In addition to cues to orientation (Taube 1998), place cell firing is strongly driven by any extended boundaries to motion within the environment. O'Keefe and Burgess (1996) recorded from the same cells across similar (rectangular) environments that differed in their dimensions. They observed that the location of peak firing of a given place cell typically remained in a constant position relative to the nearest walls, and in addition, several of the firing fields were stretched along the axes of the environment. They proposed that place cells received inputs that are tuned to respond to the presence of a barrier at a given distance along a given allocentric direction, with sharper tuning at shorter distances; this is the socalled boundary vector cell (BVG) model (Barry et al. 2006; Hartley et al. 2000), see Figure 4. The allocentric directions of BVC tuning are presumably defined relative to the head-direction cells, given that place cell and head-direction cells always seem to rotate consistently when external or internal orientation is manipulated.

In contrast to the robust effect of environmental boundaries on place cell firing, discrete landmarks within an environment have very little effect on place cell firing (Cressant et al. 1997). Equally, while removing individual distal cues to orientation does not have a marked effect on place cell firing (although the overall orientation of the representation may drift), removing environmental boundaries tends to lead to destruction of the place cell response (Barry et al. 2006; Barry et al. 2007). The BVC model has been used both to predict the pattern of place cell firing following deformation of the environmental boundaries to make an environment of different shape and size, including the addition of extended walls within the maze (Hartley et al. 2000). It has also been used to predict the search behavior of humans returning to a previously seen location, by assuming that they solve the task by moving to maximize the match between their current place cell representation and a stored place cell representation of the target location (Hartley et al. 2000; Hartley et al. 2004; O'Keefe \& Burgess 1996).

The BVC model, in combination with models of the firing properties of neurons in the parietal lobe (Pouget \& Sejnowski 1997; Salinas \& Abbott 1995) suggest a computational model of memory and imagery for spatial scenes (Byrne et al. 2007; Burgess et al. 2001b; Becker \& Burgess 2001). In this model, 


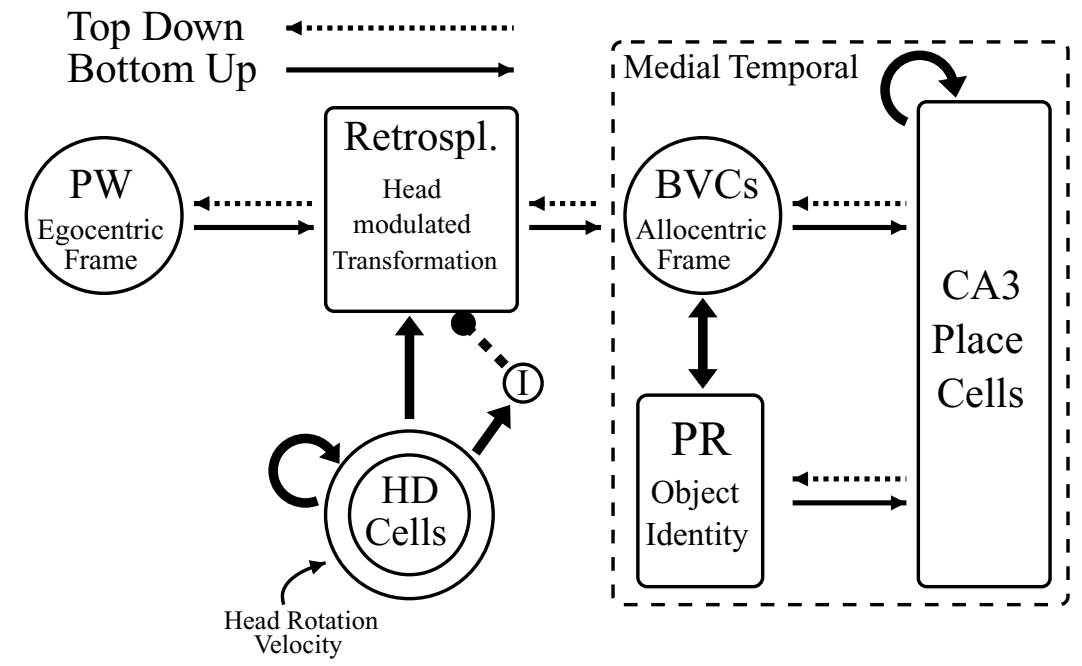

FIGURE 5. Schematic temporo-parietal model of spatial memory and imagery. Each box represents a set of neurons in a different brain region (PW: medial parietal "window" for visual perception and imagery; Retrospl: retroplenial cortex and parieto-occipital sulcus mediating ego-allo translation in conjunction with area 7a; HD cells: head-direction cells; BVCs: parahippocampal boundary vector cells; PR: perirhinal cortical encoding of visual identity). Thin solid arrows represent "bottom-up" connections from egocentric visual perception and imagery to allocentric medial temporal memory representations; thin dashed arrows represent "top-down" connections back again. Adapted from Byrne, Becker, \& Burgess (2007).

connections between hippocampal place cells, parahippocampal boundary vector cells, and perirhinal cells encoding visual textures/features form an associative memory. Thus, within a familiar environment, a partial cue can reactivate the hippocampal representation of occupying a single location within an environment, which in turn reactivates the corresponding parahippocampal and perirhinal representations of environmental boundaries and visual features respectively. To be able to examine the products of this reconstructive process in visual imagery, the allocentric (North, South, East, West) parahippocampal representation must be translated into an egocentric (left, right, ahead, behind) medial parietal representation. This is assumed to occur via processing by gain-field neurons in posterior parietal cortex and representation of the intermediate stages of translation in retrosplenial cortex/parieto-occipital sulcus, making use of the representation of head direction found along Papez's circuit to dereference allocentric directions into egocentric ones, see Figure 5.

The model is able to simulate effects found in neuropsychological and single-unit recording experiments. For example, the effect of hemi-spatial neglect in imagery following right parietal damage can be simulated, as in the famous Milan Square experiment (Bisiach \& Luzzatti 1978), in which patients could not describe the left-hand side of an imagined view of a famous piazza in their home town, whether imagining facing towards the cathedral or away from it. This is consistent with an intact medial temporal allocentric representation of the whole square along with damage to the parietal substrates of the egocentric representation or the allo-ego translation mechanism. In addition, experiments in which place cells are recorded while visual and path-integrative information are put into conflict (Gothard et al. 1996) can also be simulated.

As noted earlier in the review, it is important to bear in mind the multiple ways in which locations can be updated to accommodate self-motion. The model described above uses the translation from allocentric medial temporal representations to egocentric medial parietal representations to perform spatial updating of the egocentric locations, and translation back to the medial temporal representations to make sure the allocentric representation of self-location is also updated appropriately. However, it is also theoretically possible to directly update the allocentric representation of selflocation given self-motion information. Again, both processes may exist in parallel, with egocentric updating most useful for keeping track of small numbers of locations over short durations and allocentric updating most useful where one's position must be maintained 
relative to larger amounts of environmental information and over longer durations.

The likely importance of task demands in determining whether egocentric or allocentric mechanisms for spatial updating are used to control behavior is illustrated by comparison of the studies by King et al. (2002; King et al. 2004) with that by Shrager et al. (2007). In this first group of studies, participants saw objects within a VR arena and were subsequently tested on their memory for the objects' locations from the same viewpoint as at presentation or from a shifted viewpoint. In the studies by King et al., the new viewpoint was imposed abruptly and, when different numbers of objects were used, the trials with different list lengths were intermingled. In these studies a developmental amnesic with focal hippocampal pathology was found to be specifically impaired from the shifted viewpoint, interpreted as consistent with hippocampal support of the allocentric mechanism. In the subsequent study (Shrager et al. 2007), participants watched as the virtual arena rotated in front of them between presentation and test and performed tasks with increasing listlengths in order (i.e., spending several trials watching one location rotate, then two, then three). In this study, no specific effect of hippocampal damage upon performance from a shifted viewpoint was found, which was interpreted as an absence of evidence for hippocampal support of allocentric updating. However, a likely alternative interpretation, to my mind, is that the later study was solved by egocentric mental rotation.

It may be that the grid cells recently discovered in medial entorhinal cortex (Hafting et al. 2005) provide the neural substrate for allocentric updating of the place cell representation of one's own position within the environment, as follows. As a rat moves through its environment, each grid cell fires whenever the rat enters one of several locations which fall at the vertices of a regular triangular grid across the environment, see Figure 3. The grids of neighboring grid cells are simply shifted copies of each other, so that the relative positions of the firing locations of two grid cells remain constant across an environment and also remain constant across different environments (Fyhn et al. 2007). The nearby presubiculum, which contains head-direction cells, projects into medial (but not lateral) entorhinal cortex. This projection may allow the grid cells to perform path integration, allowing the grid cell activations to be updated in correspondence with the rat's movement. This could occur by each cell passing activity on to the appropriate neighbor (McNaughton et al. 2006; Sargolini et al. 2006; Fuhs \& Touretzky 2006) or by each cell integrating movement information individually (Burgess et al. 2007). (See the original references for the details of these proposals.) In addition, the reciprocal connections between entorhinal cortex and the hippocampus might allow the place cell and grid-cell representations to combine both motion-related inputs (to grid cells) and environmental sensory information (the BVC inputs to place cells) in determining the animal's current location (Barry et al. 2007; O'Keefe \& Burgess 2005; Burgess et al. 2007). A recent fMRI study in humans (Wolbers et al. 2007), in which they performed a path-integration task using only optic flow, showed performance to correlate with activation of the anterior hippocampus, possibly consistent with a role in allocentric spatial updating.

\section{Parallel Hippocampo-Striatal Systems in Rats}

The place cell data, summarized briefly above, seem to indicate that the hippocampus specifically processes the surface geometry of the rat's environment, with an important role also for the head-direction system, governed by distal cues to orientation. Hippocampal lesions dramatically impair performance on the classic version of the water maze, where rats must use distal landmark information as well as distance to the maze boundary to locate a hidden platform (Morris et al. 1982). Interestingly, the maze walls are powerful cues used to locate the platform even when they are transparent, illustrating the importance of continuous boundaries for navigation (Maurer \& Derivaz 2000).

Distinct hippocampal and striatal contributions to spatial navigation can be seen in tests in the water maze. Hippocampal lesions do not disrupt the ability to navigate towards a location marked by a distinct visible landmark (or "beacon"). By contrast, striatal lesions impair navigation towards a location marked by a distinct visible landmark but not to an unmarked one defined relative to distal landmarks and boundaries (Packard \& McGaugh 1992; McDonald \& White 1994). When a location is defined by its distance and direction from an intramaze cue (given distal orienting cues), and not by the maze boundary, hippocampal damage does not impair navigation (Pearce et al. 1998), although lesions of the anterior thalamus (with presumed disruption of the head-direction system) do impair navigation (Wilton et al. 2001). Thus, the hippocampus may define locations relative to the boundary, while the striatum defines locations relative to local landmarks, and the head-direction system is required to derive a heading direction from distant landmarks.

The distinct contributions of hippocampal and striatal systems to spatial cognition can also be seen in the plus maze. In this task, rats are trained in an initial learning phase to retrieve food from the end of one arm (e.g., West), starting from another arm (e.g., 
South). This paradigm can be used to elegantly study whether rats learn to navigate to the food through learning a stereotyped response (turn left) or through learning the place within the test room (presumably defined by distal cues in the environment). The use of a response or a place strategy can be assessed during a probe trial in which rats start from a novel arm (e.g., North). The rat could either follow the learned response, that is, turning left and thus searching for food in the East arm (response strategy), or follow a place strategy and search in the arm in the West of the testing room. In probe trials after 8 and 16 days of training, healthy rats shifted from approaching the "place" associated with food after 8 days to making the turn "response" associated with food after 16 days. However, injections of lidocaine to inactivate the hippocampus abolished place learning, while injections into the striatum abolish response learning (Packard \& McGaugh 1996).

We have described some of the different types of neural representation available to animals when solving spatial tasks. The head-direction system found throughout Papez's circuit may provide orientation, while the hippocampus has been identified with environment-centered representations of locations and the dorsal striatum has been associated with approach responses to a single landmark. In addition, the projections from the head-direction system to the nucleus accumbens imply that the striatal system might also allow navigation towards unmarked locations by using a visible local landmark in conjunction with external orienting cues. In the next section we consider recent evidence for a similar dissociation between right posterior hippocampal and right dorsal striatal substrates of spatial learning in the human brain.

\section{Parallel Temporo-Striatal Systems in Humans}

Study of the neural bases of large-scale navigation in humans has recently begun to take advantage of a combination of desk-top VR and functional neuroimaging. Several early studies revealed activation in parietal, retrosplenial, and parahippocampal areas as people find their way around (e.g., Aguirre \& D'Esposito 1997; Maguire et al. 1998b; Ghaem et al. 1997), but interpretation of the functions of specific subregions remained difficult. In further experiments, some patterns began to emerge. Thus parahippocampal gyrus activation may reflect sensory (Epstein \& Kanwisher 1998) or mnemonic (Janzen \& van Turennout 2004) processing of spatial scenes and perhaps the use of peripheral vision in this (Levy et al. 2001), while hippocampal activation was found to reflect navigational accuracy and striatal (caudate) activation to reflect navigational speed (Maguire et al. 1998a). Recent advances in imaging technology, more realistic environments, and more sophisticated analyses of behavior (Spiers \& Maguire 2007) have refined these interpretations further. Hartley et al. (2003) found that hippocampal activation corresponded to flexible wayfinding using new paths through previously explored environments, while striatal activation corresponded to following well-used routes (explaining the correlation with speed in the previous study). Wolbers and Buchel (2005) examined activation during the learning of a new environment and found hippocampal activation to correspond to increases in knowledge of the environmental layout, while retrosplenial activation corresponded to the absolute level of performance.

Iaria et al. (2003) adapted an elegant paradigm for identifying the use of distal cues in rat navigation for use with humans. In this task, subjects found objects in 4 arms in a virtual 8-arm maze with distal cues present around it to provide orientation. Their memory was then tested by asking them to revisit the same 4 arms again - entering the other arms counted as an error. In probe trials, the distal cues were removed during the test phase: an increase in the number of errors indicated that the subject was making use of the distal cues rather than, for example, remembering a sequence of turns. When the study was performed in an fMRI experiment, Iaria et al. found that the dependence on distal cues correlated with hippocampal activation, while distal cue-independent responding correlated with activation of the caudate nucleus. These results are consistent with hippocampal provision of an allocentric representation, requiring the distal cues, and striatal provision of route-like egocentric responses.

It seems that the hippocampal and striatal systems can act cooperatively in the context of adaptation to brain damage. Voermans et al. (2004) compared the activation provoked by remembering routes through houses (shown as video clips) between a group of patients with Huntington's disease and a group of healthy volunteers. They found reduced caudate activation corresponding to the progression of the disease (which attacks this part of the brain) in the patient group, but also increased hippocampal activation. Thus the more flexible hippocampal system may be able to take over some of the function of the striatal system in remembering routes. The extent to which the striatal system could accommodate for hippocampal damage is an interesting question for future research.

As well as the above dissociation between striatal support of overlearned route-like responses and hippocampal support of more flexible navigation, the 


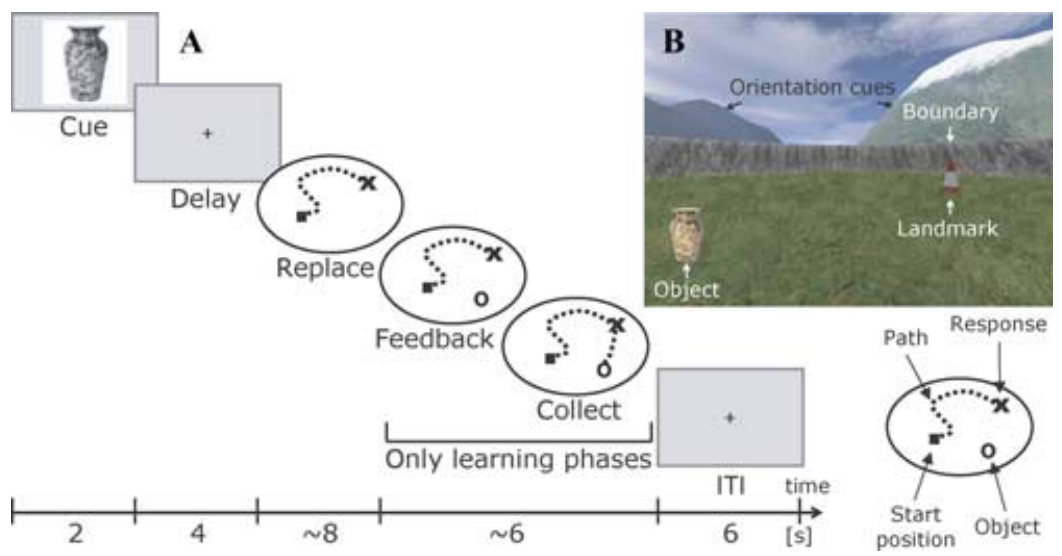

FIGURE 6. Experimental paradigm for comparing the relative contributions of local landmarks and boundaries to spatial memory. Participants play an adapted first-person perspective videogame in which they find objects within a virtual environment comprising a circular boundary and a landmark, with distal cues to orientation (rendered at infinity). (A) After collecting the objects in their respective locations, each subsequent trial has a cue phase (an object is shown) and a replace phase (the subject attempts to navigate to the object's location and presses a button). Learning trials, but not test trials, contain feedback from which subjects learn to improve their performance (i.e., replacement accuracy). Feedback consists of the object appearing in the correct location and being collected by the subject. A view of the virtual environment is shown in (B). Adapted from Doeller and Burgess (2007).

animal studies reviewed above also suggest different neural bases for processing locations relative to local landmarks and environmental boundaries. This dissociation has recently been examined in humans (Doeller et al. 2007) using a VR object-location memory task, in which, without being distinguished by any explicit instructions, some objects maintained a fixed relation to the environmental boundary while others maintained a fixed relation to a single intramaze landmark. Participants explored a VR arena bounded by a circular wall, containing a single landmark and surrounded by distant orientation cues. Within this arena they encountered four objects in four different locations, see Figure 6. On each subsequent trial they saw a picture of one of the objects (the "cue phase") and indicated its location within the arena by navigating to it from a random start location and making a button-press response (the "replace" phase). The object then appeared in its correct location and was collected (the "feedback" phase). Each set of 16 trials (four per object) composed a block, with four blocks in the entire experiment. Critically, the landmark and boundary were moved relative to each other between blocks, with two objects maintaining their location relative to the boundary and two relative to the landmark.

Performance was measured in terms of the proximity of response location to the correct location, and learning during the feedback phase could be measured as the improvement in performance on the next trial with the same object. Once the two cues had been moved relative to each other, the relative influence of landmark or boundary on responding was reflected implicitly in the distance of the response location from the locations predicted by either cue. Both cues played functionally equivalent roles in the task and were not distinguished in the participants' instructions, and their relation to the distant orientation cues remained unchanged as these were projected at infinity.

Participants gradually learned locations relative to both types of cue at similar rates, with performance increasing similarly within and across blocks (FIG. 7). Inaccurate responses largely reflected use of the incorrect cue early in each block. Consistent with the predictions from animal studies, fMRI activation in the right dorsal striatum during the feedback phase correlated with learning for landmark-related objects, while activation in the right posterior hippocampus correlated with learning for boundary-related objects. In addition, the influence of the boundary on the replacement location correlated with right posterior hippocampal activation, while the influence of the landmark correlated with right dorsal striatal activation. Thus, differential activity seen in the hippocampus and caudate corresponded to the acquisition and expression of information about locations derived from environmental landmarks or boundaries respectively.

This analysis raises the question of what distinguishes a boundary from a landmark? A simple 

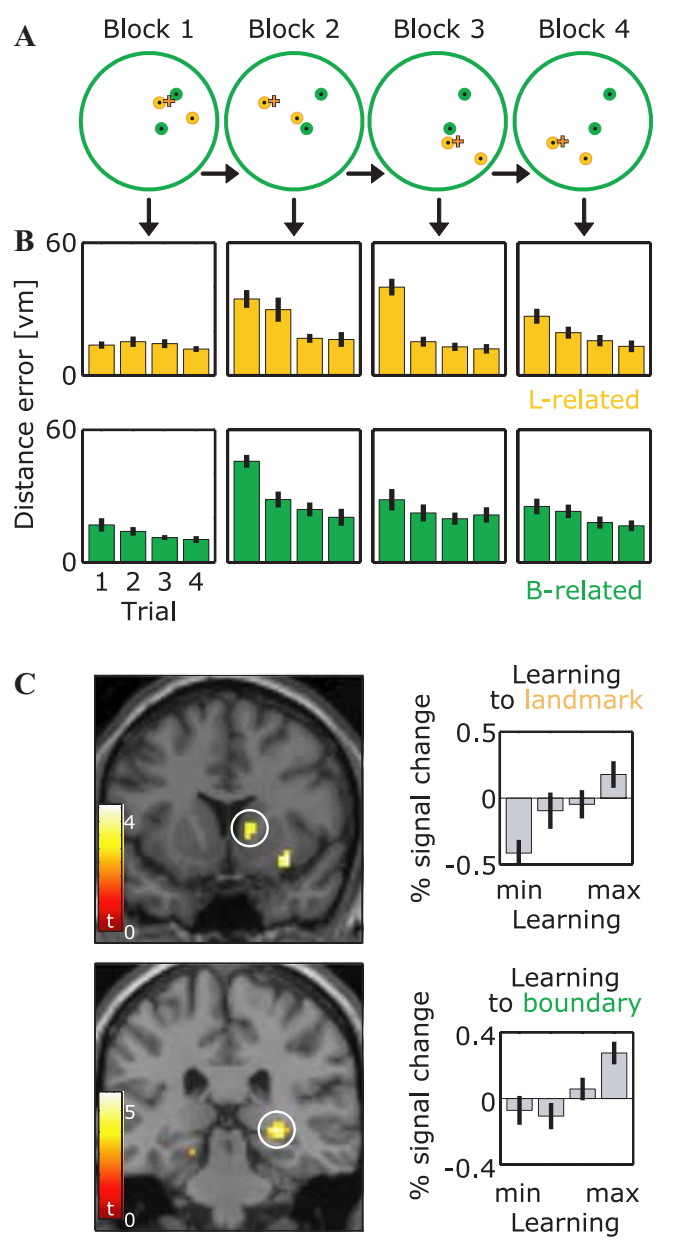

FIGURE 7. An FMRI study of the neural bases of learning locations relative to a local landmark or a local boundary; the paradigm is shown in FIGURE 6. (A) After four objects have been collected in their locations, participants completed blocks of four trials replacing each object, with feedback regarding their correct location, see FIGURE 6 . At the end of each block, the landmark and boundary were moved relative to each other, with two objects moving with the landmark (shown in orange), and two with the boundary (shown in green). (B) Object locations were learned within and between blocks from the feedback provided. Average replacement error is shown separately for the landmark-related (orange) and boundary-related (green) objects. (C) Activation during the feedback phase corresponded to learning (improvement on the next trial with that object) in the right striatum for landmark-related objects and in the right posterior hippocampus for the boundary-related objects. Adapted from Doeller, King, \& Burgess (2007).

potential answer comes from simulation of the firing of hippocampal place cells (Hartley et al. 2000; Burgess \& Hartley 2002). In these simulations, place cells effectively perform a match to the distances to the nearest obstacle, integrating over distances in all directions around the rat. Thus, for place cells, any obstacle in the environment simply has an importance proportional to the horizontal angle it subtends at the rat. This potentially provides a measure of "boundaryness" in terms of proximity and extent. However, it is not clear what aspects of environmental stimuli correspond to "landmarkness" as reflected in their influence on striatal processing.

A final consideration of this study was the nature of the interaction between the two systems in controlling behavior after they had been put into conflict by the relative motion of the two cues. In this study, the levels of activation in hippocampus and striatum could be investigated trial-by-trial as both systems contributed to a single behavior. No evidence was found of a direct interaction between activation in the two systems, other than the variation reflected in behavior. Thus it seems that both systems operate in parallel, with their activation signaling their suitability to control behavior. Such an interpretation would be consistent with the effects of lidocaine injections in rats (Packard \& McGaugh 1996) and extends previous studies of hippocampo-striatal interaction by looking at different tasks or different stages of the same task (Poldrack et al. 2001).

\section{Incidental and Reinforcement Learning Rules in Hippocampus and Striatum}

So far, I have concentrated on the representations supporting spatial cognition, but what of the rules underlying the learning of adaptive behavior? "Reinforcement learning" (Sutton \& Barto 1988) provides the dominant model for learning from feedback over multiple trials. This model, derived from the RescorlaWagner law (Rescorla \& Wagner 1972) and its development into associative learning theory (see e.g., Mackintosh 1975 and Dickinson 1980), associates cues to a measure of predicted feedback (see also, Dayan \& Abbott 2002). This rule explains results in classical conditioning and also in "instrumental conditioning," or learning to act, by also associating cues to appropriate actions. In both cases, the learning rule for adjusting the strengths of these associations depends on the difference between the predicted and actual feedback (the "prediction error"). This simple and elegant model has provided a quantitative description of a great variety of behavioral data on learning and has received strong support from evidence that the prediction error signal can be seen in the striatal targets of the dopamine system (O'Doherty et al. 2004; Pessiglione et al. 2006; Schultz 2002).

However, there has been a long-standing but unproven hypothesis that spatial learning does not obey this type of error-correcting learning rule, but rather is 
incidental, occurring independently of performance, motivation, or prediction error (Tolman 1948). This type of learning was subsequently attributed to the hippocampus by O'Keefe and Nadel (1978). However, despite the many studies since, aimed at proving this hypothesis, the results have been mixed, with most finding results consistent with reinforcement learning.

A major prediction of reinforcement learning concerns the situation where, because the association from one cue already accurately predicts feedback, there will be no prediction error and no possibility of subsequent learning to a second cue (learning is said to be "blocked"). Similarly, where learning occurs to two cues concurrently, the learning to one will be reduced by the extent to which the other accurately predicts feedback (learning to it is said to be "overshadowed").

It is possible that the confounding of hippocampal and nonhippocampal contributions to spatial cognition may have contributed to the previous findings of blocking and overshadowing in spatial tasks (Hamilton \& Sutherland 1999; Chamizo et al. 2003; Pearce et al. 2006). The above study (Doeller et al. 2007) indicates a way to dissociate the specifically hippocampal contribution to spatial learning. Under this view, learning to an environmental boundary, dependent on the hippocampus, would be incidental and would not show blocking or overshadowing, while learning to the landmark, dependent on the striatum, would conform to reinforcement learning.

Doeller and Burgess (2007) examined blocking between boundaries and landmarks within their virtual arena. Their blocking experiment consists of three phases. In a first "prelearning" phase, participants learn object locations while landmark and boundary are moved relative to each other at the beginning of each block: four objects maintaining a fixed location relative to the landmark and four other objects maintaining a fixed location relative to the boundary. In a second "compound learning" phase, both the landmark and the boundary remain in fixed positions, predicting the position of all eight objects. During the final "test" phase, memory performance is tested (without feedback) in the presence of either the landmark or the boundary alone.

If a given object is paired with cue 1 during prelearning, and the subject learns to accurately replace it on the basis of this association, then there should be little learning of the association to cue 2 during the compound learning phase. Thus performance should be poor when tested with cue 2 alone (compared to an object paired with cue 2 during prelearning). This provides a powerful test of reinforcement learning, since any effects of "learned irrelevance" or "super learning" (consequences of any association from the unpaired cue to the absence of reward) will be additive to the blocking effect.

Doeller and Burgess (2007) found that, although learning of objects to either type of cue occurred at similar rates and with similar levels of performance, there were different blocking effects for learning to the boundary and to the landmark. When tested with the landmark, performance was much worse for the object paired with the boundary during prelearning. By contrast, performance when tested with the boundary was equal for objects paired with either cue during prelearning. See FIGURE 8 for the results of the boundarylandmark blocking experiment. Consistent results were found when, again in the presence of the distal orientation cues, two landmarks were used as cues (each landmark blocking the other), when two opposing sections of the boundary were used (neither blocked the other), or when overshadowing was investigated (the boundary overshadowed the landmark, but not vice versa). Overall, the consistent finding was that learning to landmarks obeyed the predictions of reinforcement learning and learning to boundaries did not. Given the striatal and hippocampal activation corresponding to learning relative to landmarks and boundaries respectively (see above), it seems that, in this task, the striatum supports reinforcement learning relative to landmarks, while the hippocampus supports incidental learning to boundaries.

\section{Discussion: Implications beyond Spatial Cognition}

As noted in the Introduction, spatial cognition enjoys an advantage over some other fields of higher cognition in being able to share paradigms between human and animal research. This link allows some inferences regarding the actual neural representations and processes involved in human cognition to be drawn from invasive studies in animals. Given this advantage, are there more general implications that can be drawn for cognition beyond the spatial domain?

\section{Memory and Imagery: Common Processes and Neural Bases?}

Initial attempts to form a computational model of memory for spatial scenes, or for the spatial context of an event (Burgess et al. 2001b; Becker \& Burgess 2001), force a consideration of the neural mechanisms involved. I briefly review these mechanisms and then discuss their more general implications for memory and imagery (see also Hartley et al. 2007). 


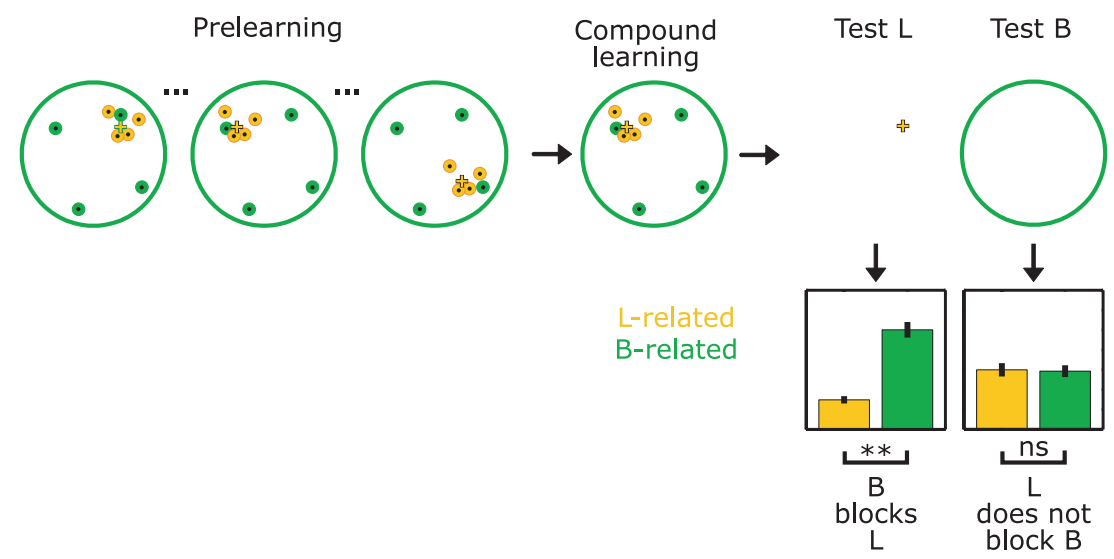

FIGURE 8. Learning to the boundary blocks learning to the landmark, but not vice versa. Participants learned eight object locations using the paradigm shown in FIGURE 6. During "prelearning" leight blocks of two trials per object on average), the landmark and boundary moved relative to each other after each block, with four objects moving with the landmark (orange " + "; object locations are orange spots) and four with the boundary (green circle, object locations are green spots). During "compound learning" (one block of six trials per object), both types of cue (landmark and boundary) remained fixed, allowing them to become associated to the locations of objects previously paired with the other cue. Both learning phases included feedback at the end of each trial. Test phases (four objects tested with the landmark L; four with the boundary B) did not include feedback and showed little learning to the landmark for objects previously associated with the boundary (Test L) but unimpeded learning to the boundary of objects previously associated with the landmark (Test B). Adapted from Doeller and Burgess (2007).

The model by Byrne and colleagues (Byrne et al. 2007) makes use of the idea that the pattern of activation of place cells is constrained by the recurrent connections within area CA3 of the hippocampus to be consistent with the subject being at a single location. Other patterns of activation, involving place cells which normally fire in different environmental locations, can only be transient, that is, the place cells form a "continuous attractor" representation of location (Zhang 1996; Samsonovich \& McNaughton 1997). The activation of place cells representing a single location can then reactivate the parahippocampal (BVC) representation of the distances and allocentric directions of environmental boundaries around that location. The retrieval of this information into visual imagery/working memory requires translation into an imaginable egocentric (head-centered) representation, involving retrosplenial/parieto-occipital sulcus and posterior parietal cortex, as well as the provision of current heading by Papez's circuit.

This model has implications for the way episodic memories or "events" are retrieved, or at least how the spatial context in which they occur is retrieved - a process thought to be specifically hippocampal dependent (Burgess et al. 2002; O'Keefe \& Nadel 1978). Thus, the hippocampus provides a strong constraint on the subsets of information retrieval from the vast amount of abstract (allocentric) knowledge stored in the sur- rounding neocortical areas. Specifically, the hippocampus restricts retrieved subsets to be mutually consistent with perception from a single location. These products of retrieval are then capable of being put into a head-centered representation for imagery in medial parietal areas via (re)constructive mechanisms in retrosplenial/parieto-occipital and posterior parietal areas, including imposition of a viewing direction onto the allocentric medial temporal representation by the head-direction cells in Papez's circuit. As well as providing an outline for the functional roles of the various regions identified in episodic memory (Burgess et al. 2001a), the model explicitly highlights the close relationship between the mechanisms and neural bases of memory and imagery (Becker \& Burgess 2001).

Recent work has verified the predicted link between memory and imagery. Similar effects of hippocampal lesions (known to affect episodic memory) have been found in spatial working memory (Hartley et al. 2007) and novel imagery (Hassabis et al. 2007). Similar patterns of activation have also been found in functional neuroimaging of memory for past events and for imagined "future" events (Addis et al. 2007). In addition, patients with hemispatial neglect in imagery, but not those with perceptual or motor neglect but unimpaired imagery, have also been found to have deficits in spatial navigation in tasks resembling the water maze (Guariglia et al. 2005). Given the close 
relationship between retrieval and imagery, and between their neural bases, it is possible that one of the hallmarks of episodic memory - that of subjective reexperience (Tulving 2001) - actually in large part reflects the success of the generation of a vivid internal image. And such imagery is of course not necessarily restricted to memory at all, although clearly implicated in much of the "reconstructive" process on which memory relies (Bartlett 1932; Addis et al. 2007).

Much future work is obviously required to identify the functional interactions between memory and imagery and between medial temporal and parietal areas, but hopefully the spatial model outlined above can provide some sort of initial framework.

\section{Learning Rules, Procedural Versus Declarative Memory, and the "Geometric Module"}

The finding of different learning rules in the processing associated with the hippocampal and striatal systems (Doeller \& Burgess 2007; Doeller et al. 2007) suggests a different way of looking at the major suggested divisions of long-term memory. Thus, it has been suggested that the acquisition of consciously retrievable long-term knowledge (whether semantic or episodic in nature) depends on the hippocampus (Scoville \& Milner 1957); this is termed "declarative" memory (Squire \& Zola-Morgan 1991), in contrast to "procedural" memory (e.g., habits, motor learning), important aspects of which depend on the striatum (Yin \& Knowlton 2006). Equally, it has been suggested that rapid one-shot encoding of events and their contexts (Tulving 1983) specifically depends on the hippocampus (Kinsbourne \& Wood 1975; O'Keefe \& Nadel 1978; Mishkin et al. 1997; Rugg \& Yonelinas 2003; Fortin et al. 2002), as opposed to slowly acquired semantic knowledge acquired slowly over multiple exposures.

These traditional dissociations might arise from differences in the basic neurobiology of the two systemsleading each system to implement a different learning rule. Thus, synaptic plasticity in the striatum may be controlled by levels of dopamine locally released (see Redgrave \& Gurney 2006) as a function of prediction error (Waelti et al. 2001; Montague et al. 2004; O'Doherty et al. 2004). By contrast, hippocampal synaptic plasticity simply reflects co-occurrence (in Doeller et al.'s study, co-ocurrence between the representation of the object and the place cell representation of location as a conjunction of bearings from the boundary).

Thus, the aspects of conscious awareness (which did not differ greatly between landmark and boundary learning) and rapidity of acquisition (which also did not differ between landmark and boundary learning) stressed by the declarative and episodic theories may be later consequences of a trial-and-error based learning rule on the one hand, and a Hebbian encoding of coincidences on the other (see also Hirsch 1974; O'Keefe \& Nadel 1978).

The neuroscience literature reviewed here demonstrates that a variety of spatial representations are available for the purposes of controlling behavior. One of these, supported by the hippocampus, is specialized for processing location relative to environmental boundaries and appears to operate a distinct, incidental, learning rule. This view of the hippocampus has curious echoes of the idea of a "geometric module" (Cheng 1986; Gallistel 1990): a module for processing the surface geometry of the surrounding environment in an "encapsulated" way (i.e., its output concerns only this subset of the information available to the animal, independent from other, e.g., featural, information). The geometric module was proposed on purely behavioral grounds, independently of some of the above ideas of hippocampal processing already present in O'Keefe and Nadel's (1978) book. The main data for the geometric module were the preferential role played by environmental boundaries in re orienting a disoriented animal (or young child; Hermer \& Spelke 1994) within a small rectangular enclosure.

The generality of the idea of a geometric module is restricted by its dependence on a single "reorientation" paradigm. For example, animals and young children do appear to be able to use featural cues to reorient in slightly different reorientation experiments, such as when using larger (Learmonth et al. 2002; Sovrano \& Vallortigara 2006), rhombic (Hupbach \& Nadel 2005), or symmetrical (Nardini et al. 2007; McGregor et al. 2004) enclosures, or learning over repeated trials (Cheng 1986; Vallortigara et al. 1990; Gouteux et al. 2001). They also routinely combine featural cues with geometric cues to navigate when not disoriented (e.g., Maurer \& Derivaz 2000), indicating that the geometric module is not strictly "encapsulated." See Cheng and Newcombe (2005) for a recent review. The conflicting patterns of results from disorientation paradigms regarding both the presence or otherwise of geometric modules and the applicability or otherwise of reinforcement learning to spatial cognition may arise in part because so many types of cue interact to determine orientation, and do so within the head-direction system (e.g., Taube 1998), rather than the hippocampus. These include local cues, distal cues, and environmental geometry, and which cues actually determine orientation depends on many factors, including each cue's apparent stability (Jeffery 
et al. 1997; Jeffery \& O'Keefe 1999), its distal or proximal location (Cressant et al. 1997), and the subject's history of disorientation (Knierim et al. 1995). By contrast, Doeller et al. (2007) attempted to dissociate a cleanly hippocampal contribution to spatial cognition, which may explain their more consistent results.

\section{Conclusions}

The field of spatial cognition has been evolving rapidly over the last few years, driven by convergence of results from the application of similar experimental paradigms in both humans and other animals. Here I have reviewed a small subset of these recent advances, focusing on the attempt to build a mechanistic understanding of how spatial behavior results from the actions of networks of neurons. In addition to the traditional strengths of cognitive science, of experimental dissociation of processes at the behavioral level, there have been vital contributions from other disciplines operating at different levels of description. These include the ability to link behavior to neural systems, for example, via neuropsychology or functional neuroimaging; the continued success of systems neuroscience, including the ability to examine the actual neural representations at play; and the ability to quantitatively integrate results at different levels by using computational modeling.

The picture which emerges of spatial cognition is one of multiple parallel systems using representations with different frames of reference, processing different aspects of the spatial scene, using different learning rules, and supported by a corresponding set of neural systems. A current challenge for the field is to begin to work out how these various neural systems, including medial temporal, parietal, and striatal regions, combine to produce the rich variety of spatial behavior underpinning our daily life. This in turn should lead to insights into the organization of other aspects of cognition and into the effects or causes of damage or dysfunction observable at the various levels of neurophysiology, systems neuroscience, and behavior.

\section{Acknowledgments}

I thank C. Bird and C.F. Doeller for commenting on this manuscript, and the UK Medical Research Council, Biotechnology and Biological Sciences Research Council and the Wayfinding project of the European Union for their support.

\section{Conflict of Interest}

The author declares no conflicts of interest.

\section{References}

Abrahams, S., Pickering, A., Polkey, C. E., \& Morris, R. G. (1997). Spatial memory deficits in patients with unilateral damage to the right hippocampal formation. Neuropsychologia, 35(1), 11-24.

Addis, D. R., Wong, A. T., \& Schacter, D. L. (2007). Remembering the past and imagining the future: Common and distinct neural substrates during event construction and elaboration. Neuropsychologia, 45(7), 1363-1377.

Aguirre, G. K., \& D'Esposito, M. (1997). Environmental knowledge is subserved by separable dorsal/ventral neural areas. 7. Neurosci., 17(7), 2512-2518.

Andersen, R. A., Essick, G. K., \& Siegel, R. M. (1987). Neurons of area 7 activated by both visual stimuli and oculomotor behavior. Exp. Brain Res., 67(2), 316-322.

Barry, C., Hayman, R., Burgess, N., \& Jeffery, K. J. (2007). Experience-dependent rescaling of entorhinal grids. Nat. Neurosci., 10(6), 682-684.

Barry, C., Lever, C., Hayman, R., Hartley, T., Burton, S., et al. (2006). The boundary vector cell model of place cell firing and spatial memory. Rev. Neurosci., 17, 71-97.

Bartlett, F. C. (1932). Remembering, a study in experimental and social psychology. New York: Macmillan.

Becker, S., \& Burgess, N. (2001). A model of spatial recall, mental imagery and neglect. Neural Information Processing Systems, 13, 96-102.

Bisiach, E., \& Luzzatti, C. (1978). Unilateral neglect of representational space. Cortex, 14, 129-133.

Bridgeman, B., Van Der Heijden, A. H. C., \& Velichkovsky, B. M. (1994). A theory of visual stability across saccadic eye movements. Behav. Brain Sci., 17, 247-292.

Burgess, N. (2006). Spatial memory: How egocentric and allocentric combine. Trends Cogn. Sci, 10(12), 551-557.

Burgess, N., Barry, C., \& O'Keefe, J. (2007). An oscillatory interference model of grid cell firing. Hippocampus, 17, 801812.

Burgess, N., Becker, S., King, J. A., \& O'Keefe, J. (2001). Memory for events and their spatial context: Models and experiments. Philos. Trans. R. Soc. Lond B Biol. Sci., 356, 1493-1503.

Burgess, N., \& Hartley, T. (2002). Orientational and geometric determinants of place and head-direction. In Neural information processing systems (Vol. 14). Cambridge, MA: MIT Press.

Burgess, N., Maguire, E. A., \& O'Keefe, J. (2002). The human hippocampus and spatial and episodic memory. $\mathrm{Neu}$ ron, 35(4), 625-641.

Burgess, N., Maguire, E. A., Spiers, H. J., \& O'Keefe, J. (2001). A temporoparietal and prefrontal network for retrieving the spatial context of lifelike events. Neuroimage, 14, 439-453.

Burgess, N., Spiers, H. J., \& Paleologou, E. (2004). Orientational manoeuvres in the dark: Dissociating allocentric and egocentric influences on spatial memory. Cognition, 94(2), 149-166.

Byrne, P., Becker, S., \& Burgess, N. (2007). Remembering the past and imagining the future: A neural model of spatial memory and imagery. Psychol. Rev., 114(2), 340 375 . 
Chamizo, V. D., Aznar-Casanova, J. A., \& Artigas, A. A. (2003). Human overshadowing in a virtual pool: Simple guidance is a good competitor against locale learning. Learn. Motiv., 34, 262-281.

Cheng, K. (1986). A purely geometric module in the rat's spatial representation. Cognition, 23(2), 149-178.

Cheng, K., \& Newcombe, N. S. (2005). Is there a geometric module for spatial orientation? Squaring theory and evidence. Psychon. Bull. Rev., 12(1), 1-23.

Colby, C. L., \& Goldberg, M. E. (1999). Space and attention in parietal cortex. Annu. Rev. Neurosci., 22, 319-349.

Cressant, A., Muller, R. U., \& Poucet, B. (1997). Failure of centrally placed objects to control the firing fields of hippocampal place cells. F. Neurosci., 17(7), 25312542.

Dayan, P., \& Abbott, L. F. (2002). Computational neuroscience. Cambridge, MA: MIT Press.

Deneve, S., Latham, P. E., \& Pouget, A. (2001). Efficient computation and cue integration with noisy population codes. Nat. Neurosci., 4(8), 826-831.

Dickinson, A. (1980). Contemporary animal learning theory. Cambridge, UK: Cambridge University Press.

Diwadkar, V. A., \& McNamara, T. P. (1997). Viewpoint dependence in scene recognition. Psychological Science, $8(4)$, 302-307.

Doeller, C. F., \& Burgess, N. (2007). Distinct error-correcting and incidental learning of location relative to landmarks and boundaries. Submitted for publication.

Doeller, C. F., King, J. A., \& Burgess, N. (2007). Parallel striatal and hippocampal systems for landmarks and boundaries in spatial memory. Submitted for publication.

Ekstrom, A. D., Kahana, M. J., Caplan, J. B., Fields, T. A., Isham, E. A., et al. (2003). Cellular networks underlying human spatial navigation. Nature, 425(6954), 184-188.

Epstein, R., \& Kanwisher, N. (1998). A cortical representation of the local visual environment. Nature, 392(6676), 598-601.

Etienne, A. S., Maurer, R., \& Seguinot, V. (1996). Path integration in mammals and its interaction with visual landmarks. F. Exp. Biol., 199(Pt 1), 201-209.

Fenton, A. A., Csizmadia, G., \& Muller, R. U. (2000). Conjoint control of hippocampal place cell firing by two visual stimuli. I. The effects of moving the stimuli on firing field positions. F. Gen. Physiol, 116(2), 191-209.

Fortin, N. J., Agster, K. L., \& Eichenbaum, H. B. (2002). Critical role of the hippocampus in memory for sequences of events. Nat. Neurosci., 5(5), 458-462.

Fuhs, M. C., \& Touretzky, D. S. (2006). A spin glass model of path integration in rat medial entorhinal cortex. 7 . Neurosci., 26(16), 4266-4276.

Fyhn, M., Hafting, T., Treves, A., Moser, M. B., \& Moser, E. I. (2007). Hippocampal remapping and grid realignment in entorhinal cortex. Nature, 446(7132), 190-194.

Galletti, C., Battaglini, P. P., \& Fattori, P. (1995). Eye position influence on the parieto-occipital area PO (V6) of the macaque monkey. Eur. 7. Neurosci, 7(12), 2486-2501.

Gallistel, R. (1990). The organization of learning. Cambridge, MA: MIT Press.

Ghaem, O., Mellet, E., Crivello, F., Tzourio, N., Mazoyer, B., et al. (1997). Mental navigation along memorized routes activates the hippocampus, precuneus, and insula. Neuroreport, 8(3), 739-744.

Gothard, K. M., Skaggs, W. E., \& McNaughton, B. L. (1996). Dynamics of mismatch correction in the hippocampal ensemble code for space: Interaction between path integration and environmental cues. F. Neurosci., 16(24), 8027 8040 .

Gouteux, S., Thinus-Blanc, C., \& Vauclair, J. (2001). Rhesus monkeys use geometric and nongeometric information during a reorientation task. F. Exp. Psychol. Gen., 130(3), 505-519.

Guariglia, C., Piccardi, L., Iaria, G., Nico, D., \& Pizzamiglio, L. (2005). Representational neglect and navigation in real space. Neuropsychologia, 43(8), 1138-1143.

Hafting, T., Fyhn, M., Molden, S., Moser, M. B., \& Moser, E. I. (2005). Microstructure of a spatial map in the entorhinal cortex. Nature, 436, 801-806.

Hamilton, D. A., \& Sutherland, R. J. (1999). Blocking in human place learning: Evidence form virtual navigation. Psychobiol., 27, 453-461.

Hartley, T., Bird, C. M., Chan, D., Cipolotti, L., Husain, M., et al. (2007). The hippocampus is required for shortterm topographical memory in humans. Hippocampus, 17 , 34-48.

Hartley, T., Burgess, N., Lever, C., Cacucci, F., \& O'Keefe, J. (2000). Modeling place fields in terms of the cortical inputs to the hippocampus. Hippocampus, 10(4), 369-379.

Hartley, T., Maguire, E. A., Spiers, H. J., \& Burgess, N. (2003). The well-worn route and the path less traveled: Distinct neural bases of route following and wayfinding in humans. Neuron, 37(5), 877-888.

Hartley, T., Trinkler, I., \& Burgess, N. (2004). Geometric determinants of human spatial memory. Cognition, 94, 39 75.

Hassabis, D., Kumaran, D., Vann, S. D., \& Maguire, E. A. (2007). Patients with hippocampal amnesia cannot imagine new experiences. Proc. Natl. Acad. Sci. U.S.A., 104(5), 1726-1731.

Hermer, L., \& Spelke, E. S. (1994). A geometric process for spatial reorientation in young children. Nature, 370(6484), 57-59.

Hirsch, R. (1974). The hippocampus and contextual retrieval of information from memory: A theory. Behav. Biol., 12(4), 421-444.

Hupbach, A., \& Nadel, L. (2005). Reorientation in a rhombic environment: No evidence for an encapsulated geometric module. Cognitive Development, 20, 279-302.

Iaria, G., Petrides, M., Dagher, A., Pike, B., \& Bohbot, V. D. (2003). Cognitive strategies dependent on the hippocampus and caudate nucleus in human navigation: Variability and change with practice. F. Neurosci., 23(13), 5945-5952.

Janzen, G., \& van Turennout, M. (2004). Selective neural representation of objects relevant for navigation. Nat. Neurosci., 7(6), 673-677.

Jeffery, K. J., Donnett, J. G., Burgess, N., \& O’Keefe, J. M. (1997). Directional control of hippocampal place fields. Exp. Brain Res., 117(1), 131-142.

Jeffery, K. J., \& O'Keefe, J. M. (1999). Learned interaction of visual and idiothetic cues in the control of place field orientation. Exp. Brain Res., 127(2), 151-161. 
King, J. A., Burgess, N., Hartley, T., Vargha-Khadem, F., \& O'Keefe, J. (2002). Human hippocampus and viewpoint dependence in spatial memory. Hippocampus, 12(6), $811-$ 820.

King, J. A., Trinkler, I., Hartley, T., Vargha-Khadem, F., \& Burgess, N. (2004). The hippocampal role in spatial memory and the familiarity-recollection distinction: A single case study. Neuropsychology, 18, 405-417.

Kinsbourne, M., \& Wood, F. (1975). Short-term memory and the amnesic syndrome. In D. Deutsch \& J. A. Deutsch (Eds.), Short term memory (pp. 257-291). New York: Academic.

Knierim, J. J., Kudrimoti, H. S., \& McNaughton, B. L. (1995). Place cells, head direction cells, and the learning of landmark stability. F. Neurosci., 15 (3 Pt 1), 1648-1659.

Kubie, J. L., Fenton, A., Novikov, N., Touretzky, D., \& Muller, R. U. (2007). Changes in goal selection induced by cue conflicts are in register with predictions from changes in place cell field locations. Behav. Neurosci., 121(4), 751763.

Learmonth, A. E., Nadel, L., \& Newcombe, N. S. (2002). Children's use of landmarks: Implications for modularity theory. Psychol. Sci., 13(4), 337-341.

Lenck-Santini, P. P., Rivard, B., Muller, R. U., \& Poucet, B. (2005). Study of CAl place cell activity and exploratory behavior following spatial and nonspatial changes in the environment. Hippocampus, 15(3), 356-369.

Levy, I., Hasson, U., Avidan, G., Hendler, T., \& Malach, R. (2001). Center-periphery organization of human object areas. Nat. Neurosci., 4, 533-539.

Mackintosh, N. J. (1975). A theory of attention: Variations in the associability of stimuli with reinforcement. Psychol. Rev., 82, 276-298.

Maguire, E. A., Burgess, N., Donnett, J. G., Frackowiak, R. S., Frith, C. D., et al. (1998a). Knowing where and getting there: A human navigation network. Science, 280(5365), 921-924.

Maguire, E. A., Burgess, N., Donnett, J. G., O’Keefe, J., \& Frith, C. D. (1998b). Knowing where things are: Parahippocampal involvement in encoding object locations in virtual large-scale space. f. Cogn. Neurosi., 10(1), 61-76.

Maurer, R., \& Derivaz, V. (2000). Rats in a transparent morris water maze use elemental and configural geometry of landmarks as well as distance to the pool wall. Spatial Cognition and Computation, 2, 135-156.

May, M. (2004). Imaginal perspective switches in remembered environments: Transformation versus interference accounts. Cognit. Psychol., 48(2), 163-206.

McDonald, R. J., \& White, N. M. (1994). Parallel information processing in the water maze: Evidence for independent memory systems involving dorsal striatum and hippocampus. Behav. Neural Biol., 61(3), 260-270.

McGregor, A., Hayward, A. J., Pearce, J. M., \& Good, M. A. (2004). Hippocampal lesions disrupt navigation based on the shape of the environment. Behav. Neurosci., 118(5), 1011-1021.

McNamara, T. P., Rump, B., \& Werner, S. (2003). Egocentric and geocentric frames of reference in memory of large-scale space. Psychon. Bull. Rev., 10(3), 589595.
McNaughton, B. L., Battaglia, F. P., Jensen, O., Moser, E. I., \& Moser, M. B. (2006). Path integration and the neural basis of the "cognitive map." Nat. Rev. Neurosci., 7(8), 663 678.

Melcher, D. (2007). Predictive remapping of visual features precedes saccadic eye movements. Nat. Neurosci., 10(7), 903-907.

Milner, A. D., Dijkerman, H. C., \& Carey, D. P. (1999). Visuospatial processing in a case of visual form agnosia. In N. Burgess, K. J. Jeffery, \& J. O'Keefe (Eds.), The hippocampal and parietal foundations of spatial cognition (pp. 443-466). Oxford, UK: Oxford University Press.

Mishkin, M., Suzuki, W. A., Gadian, D. G., \& VarghaKhadem, F. (1997). Hierarchical organization of cognitive memory. Philos. Trans. R. Soc. Lond B Biol. Sci., 352(1360), 1461-1467.

Montague, P. R., Hyman, S. E., \& Cohen, J. D. (2004). Computational roles for dopamine in behavioural control. Nature, 431(7010), 760-767.

Morris, R. G. M., Garrud, P., Rawlins, J. N., \& O'Keefe, J. (1982). Place navigation impaired in rats with hippocampal lesions. Nature, 297(5868), 681-683.

Mou, W., \& McNamara, T. P. (2002). Intrinsic frames of reference in spatial memory. 7. Exp. Psychol. Learn. Mem. Cogn., 28(1), 162-170.

Mou, W., McNamara, T. P., Valiquette, C. M., \& Rump, B. (2004). Allocentric and egocentric updating of spatial memories. 7. Exp. Psychol. Learn Mem. Cogn., 30(1), 142 157.

Muller, R. U. (1996). A quarter of a century of place cells. Neuron, 17(5), 813-822.

Muller, R. U., \& Kubie, J. L. (1989). The firing of hippocampal place cells predicts the future position of freely moving rats. F. Neurosci., 9(12), 4101-4110.

Nardini, M., Atkinson, J., \& Burgess, N. (2007). Children reorient using the left/right sense of coloured landmarks at 18-24 months. Cognition. Epub ahead of print. DOI:10.1016/j.cognition.2007.02.007.

Nardini, M., Burgess, N., Breckenridge, K., \& Atkinson, J. (2006). Differential developmental trajectories for egocentric, environmental and intrinsic frames of reference in spatial memory. Cognition, 101(1), 153-172.

O’Doherty, J., Dayan, P., Schultz, J., Deichmann, R., Friston, K., et al. (2004). Dissociable roles of ventral and dorsal striatum in instrumental conditioning. Science, 304(5669), 452-454.

O'Keefe, J. (1976). Place units in the hippocampus of the freely moving rat. Exp. Neurol., 51(1), 78-109.

O'Keefe, J., \& Burgess, N. (1996). Geometric determinants of the place fields of hippocampal neurons. Nature, 381(6581), 425-428.

O'Keefe, J., \& Burgess, N. (2005). Dual phase and rate coding in hippocampal place cells: Theoretical significance and relationship to entorhinal grid cells. Hippocampus, 15, 853-866.

O'Keefe, J., \& Conway, D. H. (1978). Hippocampal place units in the freely moving rat: Why they fire where they fire. Exp. Brain Res., 31(4), 573-590.

O'Keefe, J., \& Nadel, L. (1978). The hippocampus as a cognitive map. Oxford, UK: Oxford University Press. 
O'Keefe, J., \& Speakman, A. (1987). Single unit activity in the rat hippocampus during a spatial memory task. Exp. Brain Res., 68(1), 1-27.

Ono, T., Nakamura, K., Fukuda, M., \& Tamura, R. (1991). Place recognition responses of neurons in monkey hippocampus. Neurosci. Lett., 121(1-2), 194-198.

Packard, M. G., \& McGaugh, J. L. (1992). Double dissociation of fornix and caudate nucleus lesions on acquisition of two water maze tasks: Further evidence for multiple memory systems. Behav. Neurosci., 106(3), 439-446.

Packard, M. G., \& McGaugh, J. L. (1996). Inactivation of hippocampus or caudate nucleus with lidocaine differentially affects expression of place and response learning. Neurobiol. Learn. Mem., 65(1), 65-72.

Pearce, J. M., Graham, M., Good, M. A., Jones, P. M., \& McGregor, A. (2006). Potentiation, overshadowing, and blocking of spatial learning based on the shape of the environment. F. Exp. Psychol.Anim. Behav. Process, 32(3), 201214.

Pearce, J. M., Roberts, A. D., \& Good, M. (1998). Hippocampal lesions disrupt navigation based on cognitive maps but not heading vectors. Nature, 396(6706), 75-77.

Pessiglione, M., Seymour, B., Flandin, G., Dolan, R. J., \& Frith, C. D. (2006). Dopamine-dependent prediction errors underpin reward-seeking behaviour in humans. $\mathrm{Na}$ ture, 442(7106), 1042-1045.

Piaget, J., \& Inhelder, B. (1956). The child's conception of space. London: Routledge.

Pico, R. M., Gerbrandt, L. K., Pondel, M., \& Ivy, G. (1985). During stepwise cue deletion, rat place behaviors correlate with place unit responses. Brain Res., 330(2), 369-372.

Poldrack, R. A., Clark, J., Pare-Blagoev, E. J., Shohamy, D., Creso, M. J., et al. (2001). Interactive memory systems in the human brain. Nature, 414(6863), 546-550.

Pouget, A., \& Sejnowski, T. J. (1997). A new view of hemineglect based on the response properties of parietal neurones. Philos. Trans. R. Soc. Lond B Biol. Sci., 352(1360), 1449-1459.

Redgrave, P., \& Gurney, K. (2006). The short-latency dopamine signal: A role in discovering novel actions? Nat. Rev. Neurosci., 7(12), 967-975.

Rescorla, R. A., \& Wagner, A. R. (1972). A theory of Pavlovian conditioning: Variations in the effectiveness of reinforcement and non-reinforcement. In A. H. Black \& W. F. Prokasy (Eds.), Classical conditioning II. Current research and theory (pp. 64-99). New York: Appleton-Century-Crofts.

Rieser, J. J. (1989). Access to knowledge of spatial structure at novel points of observation. 7. Exp. Psychol.-Learn. Mem. Cogn., 15(6), 1157-1165.

Ross, J., Morrone, M. C., Goldberg, M. E., \& Burr, D. C. (2001). Changes in visual perception at the time of saccades. Trends Neurosci, 24(2), 113-121.

Rugg, M. D., \& Yonelinas, A. P. (2003). Human recognition memory: A cognitive neuroscience perspective. Trends Cogn. Sci., 7(7), 313-319.

Salinas, E., \& Abbott, L. F. (1995). Transfer of coded information from sensory to motor networks. F. Neurosci., 15(10), 6461-6474.

Samsonovich, A., \& McNaughton, B. L. (1997). Path integration and cognitive mapping in a continuous attractor neural network model. F. Neurosci., 17(15), 5900-5920.
Sargolini, F., Fyhn, M., Hafting, T., McNaughton, B. L., Witter, M. P., et al. (2006). Conjunctive representation of position, direction, and velocity in entorhinal cortex. Science, 312(5774), 758-762.

Schmidt, T., \& Lee, E. Y. (2006). Spatial memory organized by environmental geometry. Spatial Cognition \& Computation, 6(4), 347-369.

Schultz, W. (2002). Getting formal with dopamine and reward. Neuron, 36(2), 241-263.

Scoville, W. B., \& Milner, B. (1957). Loss of recent memory after bilateral hippocampal lesions. F. Neurol. Neurosurg. Psychiatry, 20, 11-21.

Shelton, A. L., \& McNamara, T. P. (1997). Multiple views of spatial memory. Psychon. Bull. Rev., 4(1), 102-106.

Shrager, Y., Bayley, P. J., Bontempi, B., Hopkins, R. O., \& Squire, L. R. (2007). Spatial memory and the human hippocampus. Proc. Natl. Acad. Sci. U.S.A., 104(8), $2961-$ 2966.

Simons, D. J., \& Wang, R. F. (1998). Perceiving real-world viewpoint changes. Psychological Science, 9, 315-320.

Snyder, L. H., Grieve, K. L., Brotchie, P., \& Andersen, R. A. (1998). Separate body- and world-referenced representations of visual space in parietal cortex. Nature, 394(6696), 887-891.

Sovrano, V. A., \& Vallortigara, G. (2006). Dissecting the geometric module: A sense linkage for metric and landmark information in animals' spatial reorientation. Psychological Science, 17(7), 616-621.

Spiers, H. J., Burgess, N., Hartley, T., Vargha-Khadem, F., \& O'Keefe, J. (2001a). Bilateral hippocampal pathology impairs topographical and episodic memory but not visual pattern matching. Hippocampus, 11, 715-725.

Spiers, H. J., Burgess, N., Maguire, E. A., Baxendale, S. A., Hartley, T., et al. (2001b). Unilateral temporal lobectomy patients show lateralised topographical and episodic memory deficits in a virtual town. Brain, 124, 2476-2489.

Spiers, H. J., \& Maguire, E. A. (2007). A navigational guidance system in the human brain. Hippocampus, 17(8), 618626.

Squire, L. R., \& Zola-Morgan, S. (1991). The medial temporal lobe memory system. Science, 253(5026), 1380 1386.

Sutton, R. S., \& Barto, A. G. (1988). Reinforcement learning: An introduction. Cambridge, MA: MIT Press.

Taube, J. S. (1998). Head direction cells and the neuropsychological basis for a sense of direction. Prog. Neurobiol., 55, 225-256.

Tolman, E. C. (1948). Cognitive maps in rats and men. Psychol. Rev., 55, 189-208.

Tulving, E. (1983). Elements of episodic memory. Oxford, UK: Clarendon Press.

Tulving, E. (2001). Episodic memory and common sense: How far apart? Philos. Trans. R. Soc. Lond. B Biol. Sci., 356(1413), 1505-1515.

Vallortigara, G., Zanforlin, M., \& Pasti, G. (1990). Geometric modules in animals' spatial representations: A test with chicks (Gallus gallus domesticus). F. Comp. Psychol., 104(3), 248-254.

Voermans, N. C., Petersson, K. M., Daudey, L., Weber, B., Van Spaendonck, K. P., et al. (2004). Interaction between 
the human hippocampus and the caudate nucleus during route recognition. Neuron, 43(3), 427-435.

von Helmholtz, H. (1866). Handbuch der physiologischen optik. Hamburg, Germany: Leopold Voss.

Waelti, P., Dickinson, A., \& Schultz, W. (2001). Dopamine responses comply with basic assumptions of formal learning theory. Nature, 412(6842), 43-48.

Waller, D., \& Hodgson, E. (2006). Transient and enduring spatial representations under disorientation and selfrotation. 7. Exp. Psychol. Learn Mem. Cogn., 32(4), 867882.

Wang, R. F., \& Simons, D. J. (1999). Active and passive scene recognition across views. Cognition, 70(2), 191-210.

Wang, R. F., \& Spelke, E. (2000). Updating egocentric representations in human navigation. Cognition, 77, 215-250.

Wang, R. F., \& Spelke, E. S. (2002). Human spatial representation: Insights from animals. Trends Cogn. Sci., 6(9), 376-382.

White, N. M., \& McDonald, R. J. (2002). Multiple parallel memory systems in the brain of the rat. Neurobiol. Learn Mem., 77(2), 125-184.

Wilton, L. A., Baird, A. L., Muir, J. L., Honey, R. C., \& Aggleton, J. P. (2001). Loss of the thalamic nuclei for "head direction" impairs performance on spatial memory tasks in rats. Behav. Neurosci., 115(4), 861-869.

Wolbers, T., \& Buchel, C. (2005). Dissociable retrosplenial and hippocampal contributions to successful formation of survey representations. F. Neurosci., 25(13), 3333-3340.

Wolbers, T., Wiener, J. M., Mallot, H. A., \& Buchel, C. (2007). Differential recruitment of the hippocampus, medial prefrontal cortex, and the human motion complex during path integration in humans. F. Neurosci., 27(35), 9408-9416.

Wraga, M., Creem, S. H., \& Proffitt, D. R. (2000). Updating displays after imagined object and viewer rotations. f. Exp. Psychol. Learn. Mem. Cogn., 26(1), 151-168.

Yin, H. H., \& Knowlton, B. J. (2006). The role of the basal ganglia in habit formation. Nat. Rev. Neurosci., 7(6), 464 476.

Zhang, K. (1996). Representation of spatial orientation by the intrinsic dynamics of the head-direction cell ensemble: A theory. F. Neurosici, 16(6), 2112-2126.

Zipser, D., \& Andersen, R. A. (1988). A back-propagation programmed network that simulates response properties of a subset of posterior parietal neurons. Nature, 331(6158), 679-684. 